\title{
Exclusive Breastfeeding Practice in Indonesia: A Population-Based Study
}

\author{
Maria Gayatri* \\ Family Planning Research and Development Unit, National Population and Family Planning Board, East Jakarta, Indonesia
}

Background: It is a well-known fact that exclusive breastfeeding benefits both mothers and their babies. The aim of this study is to assess the associated factors influencing exclusive breastfeeding of babies until 6 months of age. Methods: The study used data from the 2017 Indonesia Demographic and Health Survey. Overall, 1,542 women who had infants aged below 6 months were included in the study. Sociodemographic and maternal health service utilization factors were examined for association with exclusive breastfeeding, using logistic regression for a complex sample design.

Results: The proportion of exclusive breastfeeding was 52.3\% (95\% confidence interval [CI], 0.498-0.548). Parity, antenatal care visits, early initiation of breastfeeding, low-income households, and rural areas were significant factors associated with the increased likelihood of exclusive breastfeeding. However, working status (adjusted odds ratio [aOR], 0.70; 95\% CI, 0.57-0.86) and caesarean delivery or C-section (aOR, 0.75; 95\% CI, 0.57-0.99) were factors for a lower likelihood of exclusive breastfeeding.

Conclusion: Working women are at risk of discontinuation of exclusive breastfeeding. Mothers who had a normal vaginal delivery and practiced early initiation of breastfeeding had a higher rate of exclusive breastfeeding. These findings have important implications for developing comprehensive guidance and resources for women regarding the importance of exclusive breastfeeding during the early postpartum period.

Keywords: Exclusive Breastfeeding; Infant; Child Health; Indonesia

Received: June 4, 2020, Revised: July 25, 2020, Accepted: July 30, 2020

*Corresponding Author: Maria Gayatri https://orcid.org/0000-0002-2792-5586

Tel: +62-81382580297, Fax: +62-21-8008554,E-mail: maria.gayatri.bkkbn@gmail.com, maria.gayatri@bkkbn.go.id 


\section{INTRODUCTION}

Breastmilk contains nutritional components that are vital for infant health, growth, and development. ${ }^{1)}$ Breastfeeding is a major public health intervention that is associated with a reduction in respiratory and gastrointestinal morbidity, ${ }^{2-5)}$ reducing the risk of infections ${ }^{5-7)}$ and lowering the risk of child mortality. ${ }^{2,6,8)}$ Exclusive breastfeeding (EBF) can protect babies from gastrointestinal infections, which can cause severe nutrient depletion. ${ }^{6}$ The first fluid when a mother starts breastfeeding is called colostrum, which is a rich source of immunological as well as developmental components.

Breastfeeding also benefits mothers by helping the uterus return to normal size after delivery, while helping them develop a close bond with the baby, increase birth spacing, and reduce the risk of breast and ovarian cancers. ${ }^{1,6)} \mathrm{EBF}$ is considered an effective method of contraception and is characterized as lactational amenorrhea method, protecting against the risk of pregnancy.

There are many factors influencing successful EBF such as maternal age, ${ }^{9-11)}$ parity, ${ }^{9,10,12,13)}$ employment status, ${ }^{1,12,14)}$ and place of residence. ${ }^{15-17)}$ Some other factors that are related to maternal health service utilization also play a significant role in promoting EBF. Knowledge, attitude, and the communication skills of health providers are important to motivate mothers to practice $\mathrm{EBF}^{1)}$ as are the mode of delivery, ${ }^{1)}$ antenatal care (ANC) visit, ${ }^{18)}$ and the practice of early initiation of breastfeeding (EIBF). ${ }^{19)}$

This research utilized data from the latest version of the Indonesia Demographic and Health Survey (IDHS) 2017, and used detailed determinants including sociodemographic and maternal health service utilization factors such as women's working status. ANC visits are important predictors in this research. The primary objective of this study is to assess the associated factors of EBF among babies until 6 months of age.

\section{METHODS}

\section{Sample and Procedures}

Data for this cross-sectional study was taken from IDHS 2017, which is a nationally representative survey conducted every 5 years that uses a multistage probability sampling design. In the first stage, census blocks were selected with systematic sampling proportional and stratified by urban and rural areas. In the second, eight households were selected systematically among 25 normal households in each selected census block. ${ }^{20)}$ All the respondents provided informed consent before starting the interview. Training of field staff was conducted in order to have the same understanding in the survey's management and each point in the questionnaire, including operational definitions.

IDHS has been designed to collect data on marriage and sexual activity, fertility, family planning, infant and child mortality, maternal health care, child health, infant and young child feeding practices, human immunodeficiency virus (HIV)/human immunodeficiency virusrelated knowledge, women's empowerment, and father's participation in family health care. The information on breastfeeding was obtained through interviews with mothers. The data related to IDHS were also available and free to download after a simple registration process on the demographic and health survey website (https://dhsprogram. com/data).

Eligible women, who met the inclusion criteria, were enrolled in the analysis. The details of respondent selection are shown in Figure 1. The inclusion criteria in this research were: (1) mothers who delivered in the past 2 years preceding the survey; (2) the infant was alive; (3) the infant was the youngest child; (4) the infant lived with the mother; and (5) the infant was under 6 months of age. The exclusion criteria were: (1) infants who suffered from acute or chronic illnesses; (2) infants who were delivered prematurely; (3) infants of multiple births such as twins, triplets, quadruplets, etc.; (4) infants under 6 months of age whose mothers refused to sign the informed consent form; (5) mothers of infants with special dietary requirements or feeding difficulties; and (6) mothers and children aged 0-23 months who were HIV exposed or infected. The respondents with exclusion criteria were not eligible to participate in this study. Based on the inclusion and exclusion criteria, the respondents involved in this analysis totaled 1,542 women.

\section{Outcomes}

We examined the practice of breastfeeding among infants under 6 months of age in the specific group of EBF and non-EBF mothers. In this study, EBF was defined as infant feeding with only breastmilk and no additional food or drink supplements for the first 6 months of life. The outcome variable was defined in a binary form, (1) non-EBF and (2) EBF. The dependent variable was calculated through certain steps. The first step, mothers reported that they had the youngest child in the last 2 years and the child lived with the mother. The second step, mothers reported that their child was being breastfed. The third step, mothers reported that they gave external food in addition to breastmilk to the child such as water (plain water or sugar water), other liquids (juice, tea, or coffee), milk (tinned or powdered milk, fresh milk,

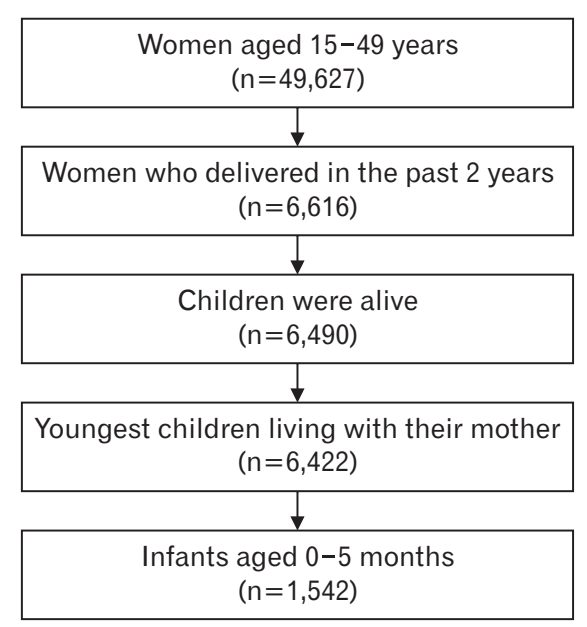

Figure 1. Respondent selection. 
baby formula), and solid food (fortified baby food, porridge/gruel, soup/clear broth). The outcome would be categorized as EBF and coded as 1 if the youngest child lived with the mother and was fully breastfed without any complementary feeding. Furthermore, mothers who did not breastfeed or mothers who breastfed but gave other complementary feeding were coded as 0 , namely non-EBF.

\section{Predictors}

The independent variables included sociodemographic and maternal health service utilization factors that might be associated with the practice of EBF. The sociodemographic factors consisted of the mother's age, parity, mother's occupation, wealth index of the household, and place of residence. Mother's age was categorized into age groups 15-19 years, 20-29 years, and 30 years or above. Parity was classified into 1, 2-3, and 4 or more. Mother's occupation was categorized into non-working and working (such as professional, technical, managers and administration, clerical, sales, service, agricultural worker or industrial worker). Place of residence was classified into rural and urban.

In IDHS 2017, the household wealth index was organized into five wealth quintiles: poorest, poorer, middle, richer, and richest. These quantiles were computed based on household ownership of assets such as television, radio, telephone, refrigerator, two and four wheelers, materials used for housing construction such as flooring materials, rooms used for sleeping, place for cooking and cooking fuel, source and treatment of drinking water, and sanitation facilities. These scores were calculated using principal component analysis. The household score of each member was compiled, and each person was ranked by their score then the household score is divided into five equal categories that form the five $20 \%$ sections. The first $20 \%$ of household score was named as lowest/poorest, the second $20 \%$ was second/poorer, the third $20 \%$ was middle, the fourth $20 \%$ was fourth/richer, and the fifth $20 \%$ was highest/richest. ${ }^{20,21)}$ In this study, the quintiles of poorer and poorest were combined into poor, and richer and richest into rich. With regard to the wealth index factor score in this study, poor households had a wealth index factor score between -413,300 and 724, middle households between 1,255 and 49,231, and rich households between 49,475 and 245,764. Wealth index factor score for each respondent was already calculated and given by the Demographic and Health Survey Wealth Index.

Maternal health service utilization factors consisted of ANC visits, mode of delivery and EIBF. In the survey, women were asked the frequency of ANC visits they had done before delivery. The visits were categorized into one-four visits and five visits or more.

\section{Statistical Methods}

Descriptive analysis was used to describe the sample characteristics using frequencies and percentages. The missing values on breastfeeding practice were handled as not currently breastfeeding. ${ }^{22)}$ There were two respondents who did not report their breastfeeding practice but declared that they gave complementary food to their infants. Furthermore, missing values on complementary food or drink such as plain water, non-milk liquids (sugar water, juice, tea or coffee), other milk (baby formula, fresh milk, powdered milk), or complementary food (porridge, fortified baby food, soup, bread, noodles, eggs, fruits, meat, fish, cheese, etc.) were considered as not given. ${ }^{22)}$ In this study, there were 10 missing values of feeding the child plain water, 10 missing values of feeding baby formula, 11 missing values for non-milk liquid, and 11 missing values for complementary food. All the missing values on foods given were treated as not given. ${ }^{22}$

The use of sampling weights was required for analyzing IDHS data. Logistic regression models were used to identify the risk factors that were associated with EBF across this 6-month period. Bivariate logistic regression was used to examine the significant differences in the practice of EBF among categories in each group.

Subsequently, we used multivariate logistic regression to estimate adjusted odds ratio (aOR). Multivariate analysis was important to measure the relationship between sociodemographic and maternal health service utilization factors and the practice of EBF among mothers who had infants under 6 months of age in Indonesia. By this analysis, the influence of each factor on the outcome would be estimated by controlling for multiple simultaneous factors. Variables with probability value (P-value) less than $5 \%$ were considered as significant. The age variable was included in the multivariable model regardless of its significance considering its theoretical relevance to the practice of EBF.

All analyses were conducted using a complex sample design, including the complex samples logistic regression which may perform binary dependent variables for samples drawn by multiple probability sample. For the complex sample design, the "svy" command prefix was used in Stata ver. 15.0 (Stata Corp., College Station, TX, USA) to adjust for design effects and non-response rate.

\section{Ethics Approval}

This study was based on secondary analysis of IDHS 2017. Personal information such as names and addresses of the mothers were not included while downloading the dataset. Therefore, institutional review board approval was not required for this study.

\section{RESULTS}

Table 1 demonstrates the sociodemographic characteristics of mothers. The mean age of mothers was $28.71 \pm 6.31$ years. Based on the age interval, about $49 \%$ of mothers were aged between 20 to 29 years. Of the total 1,542 mothers, the majority were not working (59\%), had 2-3 children (56\%), and lived in rural areas (54\%). Relatively disadvantaged households were represented by $42 \%$ of respondents from poor households. More than three-fourths of mothers had ANC visits 5 times or more. More than $50 \%$ of mothers had practiced EIBF within the first hour of delivery. The highest number of mothers had normal vaginal delivery $(82 \%)$.

Despite the many benefits of EBF, only $52.3 \%$ ( $95 \%$ confidence interval [CI], 0.498-0.548) of infants under 6 months of age in Indonesia were exclusively breastfed in 2017. EBF was found to be higher among 
Table 1. Characteristics of women who had infants under 6 months, Indonesia 2017

\begin{tabular}{|c|c|c|c|}
\hline Characteristic & Non-exclusive ( $\mathrm{n}=735$ ) & Exclusive $(\mathrm{n}=807)$ & Total $(n=1,542)$ \\
\hline \multicolumn{4}{|l|}{ Mother's age (y) } \\
\hline $15-19$ & $52(7.02)$ & $54(6.70)$ & $106(6.85)$ \\
\hline $20-29$ & $347(47.25)$ & $404(50.05)$ & $751(48.72)$ \\
\hline$\geq 30$ & $336(45.73)$ & $349(43.25)$ & $685(44.43)$ \\
\hline \multicolumn{4}{|l|}{ Parity } \\
\hline 1 & 247 (33.61) & $222(27.53)$ & $469(30.43)$ \\
\hline $2-3$ & $394(53.61)$ & $471(58.30)$ & $865(56.07)$ \\
\hline$\geq 4$ & $94(12.78)$ & $114(14.16)$ & $208(13.51)$ \\
\hline \multicolumn{4}{|l|}{ Occupation* } \\
\hline Non-working & $398(54.20)$ & $514(63.72)$ & $913(59.18)$ \\
\hline Working & $337(45.80)$ & $293(36.28)$ & $629(40.82)$ \\
\hline \multicolumn{4}{|l|}{ Wealth* } \\
\hline Poor & $258(35.09)$ & 391 (48.44) & $649(42.07)$ \\
\hline Middle & $187(25.46)$ & $136(16.86)$ & $323(20.96)$ \\
\hline Rich & $290(39.44)$ & $280(34.71)$ & $570(36.96)$ \\
\hline \multicolumn{4}{|l|}{ Place of residence } \\
\hline Urban & $383(52.10)$ & $333(41.25)$ & $716(46.42)$ \\
\hline Rural & $352(47.90)$ & $474(58.75)$ & $826(53.58)$ \\
\hline \multicolumn{4}{|l|}{ ANC visit (times) } \\
\hline $1-4$ & $188(25.63)$ & 179 (22.22) & $368(23.84)$ \\
\hline$\geq 5$ & $547(74.37)$ & $628(77.78)$ & $1,174(76.16)$ \\
\hline \multicolumn{4}{|c|}{ Early initiation of breastfeeding } \\
\hline No & $391(53.21)$ & $338(41.85$ & $729(47.27)$ \\
\hline Yes & $344(46.79)$ & $469(58.15$ & $813(52.73)$ \\
\hline \multicolumn{4}{|l|}{ Mode of delivery } \\
\hline Normal vaginal & $572(78.00)$ & $688(85.25)$ & $1,260(81.73)$ \\
\hline Caesarean & $163(22.14)$ & $119(14.75)$ & $282(18.27)$ \\
\hline
\end{tabular}

Values are presented as number (\%). These values were computed by the author from the Indonesia Demographic and Health Survey 2017 data.

*The working status and wealth index are arbitrary. Both variables show the condition at the time of the survey (interview).

mothers who had five or more ANC visits and had delivered their child via normal vaginal delivery. Likewise, women who practiced EIBF tended to be successful in practicing EBF as a continuation of EIBF. EBF prevalence was higher in rural areas compared to urban areas. The EBF practice rates among working and non-working mothers were $36 \%$ and $64 \%$, respectively.

Table 2 presents the binary logistic regression estimates of odds ratio (OR) and the multivariate logistic regression estimates of aOR for selected variables of mothers with infants below 6 months of age on the practice of EBF. Based on multivariate analysis, mothers who had twothree children were more likely to practice EBF compared to those who had only one child (aOR, 1.34; 95\% CI, 1.02-1.76). Furthermore, mothers with four or more children were also more likely to exclusively breastfeed compared with single parous mothers (aOR, 1.51; 95\% CI, 1.00-2.28). Based on the wealth index, only mothers from the middle quantile were less likely to report practicing EBF (aOR, 0.51; 95\% CI, 0.39-0.69) compared with mothers from poor households. Working mothers were less likely to practice $\mathrm{EBF}$ (aOR, 0.70; 95\% CI, 0.57-0,86) compared to their counterparts who have no occupation. However, no significant difference was observed across mother's age.

The odds of mothers who had five or more ANC visits were $45 \%$ (aOR, 1.45; 95\% CI, 1.12-1.88) more likely to practice EBF compared to mothers who only had one-four visits. Among the mode of delivery, mothers who delivered via C-section were less likely to practice $\mathrm{EBF}$ (aOR, 0.75; 95\% CI, 0.57-0.99) compared to those who delivered their babies via normal vaginal delivery. Additionally, mothers who practiced EIBF were $49 \%$ (aOR, 1.49; 95\% CI, 1.21-1.84) more likely to be successful in EBF compared to those who did not practice EIBF within the first hour after delivery.

\section{DISCUSSION}

Breastfeeding has been considered as the most effective and efficient strategy to reduce the risk of infant mortality. Based on guidelines from the World Health Organization (WHO), the rating of EBF among babies aged $0-<6$ months was classified into four groups: poor (EBF percentage is $0 \%-11 \%$ ), fair (EBF percentage is $12 \%-49 \%$ ), good (EBF percentage is $50 \%-89 \%$ ), and very good (EBF percentage is $90 \%-100 \%$ ). Based on the above guidelines, the EBF rate in Indonesia (52.3\%; 95\% CI, 0.498-0.548) has been categorized as "good". However, based on data from the United Nations Children's Fund, the EBF percentage in Indonesia is still lower compared to the EBF percentage in Cambodia (65.2\%), Bangladesh (65\%), and India (58\%). ${ }^{23)}$ The Indonesian Government had published the "Government Regulation Number 33 Year 2012 on Exclusive Breastfeeding". Under Article 13, it is mandatory for health providers and health facilities to provide information and edu- 
Table 2. OR and aOR with $95 \% \mathrm{Cl}$ of explanatory variables for EBF among mothers with infants under 6 months, Indonesia 2017, obtained from logistic regression model

\begin{tabular}{|c|c|c|c|c|}
\hline \multirow{2}{*}{ Covariates } & \multicolumn{4}{|c|}{ EBF } \\
\hline & OR (95\% Cl) & P-value & $\mathrm{aOR}(95 \% \mathrm{Cl})$ & $P$-value \\
\hline \multicolumn{5}{|l|}{ Mother's age (y) } \\
\hline \multicolumn{5}{|l|}{$15-19$} \\
\hline $20-29$ & $0.91(0.74-0.99)$ & 0.055 & $0.92(0.59-1.44)$ & 0.713 \\
\hline$\geq 30$ & $0.79(0.66-0.89)$ & 0.066 & $0.74(0.45-1.21)$ & 0.232 \\
\hline \multicolumn{5}{|l|}{ Parity } \\
\hline \multicolumn{5}{|l|}{1} \\
\hline $2-3$ & $1.33(1.06-1.66)$ & 0.014 & $1.34(1.02-1.76)$ & 0.034 \\
\hline$\geq 4$ & $1.35(0.97-1.88)$ & 0.071 & $1.51(1.00-2.28)$ & 0.050 \\
\hline \multicolumn{5}{|l|}{ Occupation } \\
\hline \multicolumn{5}{|l|}{ Non-working } \\
\hline Working & $0.67(0.55-0.83)$ & 0.000 & $0.70(0.57-0.86)$ & 0.001 \\
\hline \multicolumn{5}{|l|}{ Wealth } \\
\hline \multicolumn{5}{|l|}{ Poor } \\
\hline Middle & $0.48(0.37-0.63)$ & 0.000 & $0.51(0.39-0.69)$ & 0.000 \\
\hline Rich & $0.64(0.51-0.80)$ & 0.000 & $0.78(0.60-1.02)$ & 0.068 \\
\hline \multicolumn{5}{|l|}{ Place of residence } \\
\hline \multicolumn{5}{|l|}{ Urban } \\
\hline Rural & $1.55(1.27-1.89)$ & 0.000 & $1.38(1.10-1.73)$ & 0.005 \\
\hline \multicolumn{5}{|l|}{ ANC visit (times) } \\
\hline \multicolumn{5}{|l|}{ 1-4 times } \\
\hline$\geq 5$ & $1.21(1.05-1.53)$ & 0.016 & $1.45(1.12-1.88)$ & 0.005 \\
\hline \multicolumn{5}{|c|}{ Early initiation of breastfeeding } \\
\hline \multicolumn{5}{|l|}{ No } \\
\hline Yes & $1.58(1.29-1.93)$ & 0.000 & $1.49(1.21-1.84)$ & 0.000 \\
\hline \multicolumn{5}{|l|}{ Mode of delivery } \\
\hline \multicolumn{5}{|l|}{ Normal vaginal } \\
\hline Caesarean & $0.61(0.47-0.79)$ & 0.000 & $0.75(0.57-0.99)$ & 0.041 \\
\hline
\end{tabular}

These values were computed by the author from the Indonesia Demographic and Health Survey 2017 data.

$\mathrm{OR}$, odds ratio; aOR, adjusted odds ratio; $\mathrm{Cl}$, confidence interval; EBF, exclusive breastfeeding.

cation on EBF to mothers (and their families) during ANC visits until the end period of EBF. The "Ministry of Health Regulation Number 15 Year 2014" also states that health providers, health professionals, health education providers, and manufacturers or distributors of infant formula are subject to administrative sanctions if they hinder the success of the EBF Program.

$\mathrm{EBF}$ is the key driver during the first 6 months of an infant's life. Ensuring that all women have access to information and education to EBF is a high priority to achieve optimal growth, development, and the health of children. The WHO has recommended continuing breastfeeding with complementary feeding from 6 months to up to 2 years or beyond. ${ }^{24)}$

Significant associations of sociodemographic factors such as parity, employment status, household wealth index, and place of residence have been determined in this study. The results of this study are similar to other research that multiparity was positively associated with the practice of $\mathrm{EBF}^{9,10,13,25)}$ Based on this study, the experiences and the knowledge regarding the benefits of EBF play an important role in promoting the practice. Mothers are more likely to have self confidence in breastfeeding from previous EBF experiences. ${ }^{9)}$ Contrarily, a study in India showed that first-birth order are more likely to be exclusively breastfed compared to higher birth order. ${ }^{26)}$

The results regarding non-working mothers is in line with previous studies that found a positive association between non-working mothers and the practice of $\mathrm{EBF}^{7,10,13,16,27)}$ This can be attributed to nonworking mothers getting to stay longer with their infants, so as to enable the practice of EBF frequently. ${ }^{7,13}$ However, working mothers have been associated with shorter duration of EBF and failure to practice $\mathrm{EBF}^{16)}$ Working mothers tend to be separated from their babies because they have to get back to work. A qualitative study in Depok, Indonesia, found that after returning to their occupation, some mothers believe that their breastmilk production is decreasing due to the impact of increasing stress in the workplace. ${ }^{12)}$ Working mothers face some challenges in sustaining EBF such as conflict of commitment in the workplace, limited support in the workplace, and lack of breastfeeding facilities. ${ }^{14)}$ Working women should struggle to balance their work and family life.

Our findings suggest that it is important to provide targeted breastfeeding support for working women. Workplaces should provide a private and safe place (such as lactation room) for pumping, the equipment necessary for breastmilk preservation, and breastfeeding break. Moreover, previous studies have shown that longer maternity leave 
contributed to longer duration of EBF among working mothers who have infants aged under 6 months. ${ }^{17,28-31)}$ Systematic reviews have also shown similar results of positive association of maternity leave on breastfeeding practice. ${ }^{32)}$ However, according to Article 82 of the "Indonesian Labor Law Number 13 Year 2013", maternity leave is limited to only 3 months and it is divided into 1.5 months before and 1.5 months after delivery. This regulation makes the practice of EBF for 6 months after delivery challenging for working mothers in Indonesia. Therefore, it is recommended to strengthen the existing regulation by extending maternity leave in order to facilitate EBF in the workplace.

The results reveal that mothers from lower socio-economic groups are more likely to practice EBF than those from middle-income families, which is similar to other studies..$^{10,13,17,26,27)}$ The possible explanation is that the poor have limited resources to purchase other alternative infant feeding, so breastfeeding is the only option for their infants. ${ }^{27)}$ Furthermore, high-income households have access to better education and greater opportunity for professional occupation. ${ }^{26)} \mathrm{Em}$ ployed mothers are less likely to practice EBF, especially when they do not receive support from the workplace to breastfeed optimally. ${ }^{26)}$ However, this study is inconsistent with a study conducted in Somaliland that stated the positive impact of income on EBF, considering that high income households can improve exposure to various media and have a better knowledge of $\mathrm{EBF}^{18)}$

The current study demonstrated that rural residency was associated with increased odds of EBF, which is consistent with previous studies. ${ }^{10,15,16)}$ In contrast, a study from Western India revealed that residing in rural areas was associated with non-EBF. ${ }^{26)}$ Another study in Malawi reported no significant association between place of residence and $\mathrm{EBF}^{25)}$

Mothers who delivered via C-section were less likely to practice EBF compared to mothers who had spontaneous vaginal delivery. ${ }^{1,7,9,13)}$ The lower rate of EBF among mothers who underwent C-section was related to the diminished practice of EIBF compared to mothers who delivered via normal vaginal delivery. ${ }^{1)}$ The other possible explanation may be associated with the pain and discomfort of breastfeeding after a C-section. ${ }^{7,9)}$

In this study, ANC visits were positively associated with EBF. A similar finding was found in previous studies. ${ }^{18,26)}$ The information and education provided by health care workers during ANC visits has improved the practice of EBF. An exploratory study among Swedish women suggests involving husbands in counselling sessions on breastfeeding during ANC visits, so that mothers can experience satisfaction with the family's support, which could strengthen their confidence to continue breastfeeding for 6 months. ${ }^{33)}$ Having an unsupportive partner was associated with the early discontinuation of $\mathrm{EBF}^{11)}$ A study from Sweden found the information which mothers want to know during ANC visits are the physiology of breastfeeding, signs of sufficient milk supply, and ways to increase breast milk supply. ${ }^{33)} \mathrm{A}$ qualitative study in Bhutan showed that one of the reasons why mothers use infant formula is the perception that they are not producing enough milk. ${ }^{31)}$ Therefore, counselling during ANC visits is important to improve confidence and positive perception on breastfeeding experience.

Furthermore, the practice of EIBF has a strong influence on the practice of EBF. This finding is similar to a previous study. ${ }^{19)}$ Individual attitudes in practicing initiation into breastfeeding is associated with intentions for breastfeeding. ${ }^{34)}$ EIBF occurs during the critical time after birth especially for women who are experiencing motherhood for the first time.

The findings have important implications for health providers, as it shows that mothers who deliver via C-section were less likely to practice EBF. Therefore, it is imperative to provide information about the benefits and management of breastfeeding. For working women, it is important to understand how to breastfeed appropriately and maintain lactation especially when they are separated from their infants for some hours or days. These steps are consistent with the WHO guideline in "Ten steps to successful breastfeeding". A meta-analysis on breastfeeding interventions showed that the health system and community intervention by promoting EBF, counselling, and education are more effective to promote the practice of EBF compared to personal counselling. ${ }^{15)}$ The intervention of Baby Friendly Hospital was significantly effective in increasing the rates of EBF, especially after a C-section. ${ }^{11,15)}$

The strength of this study is the large sample size and the national representative sample procedures which enable to generalize the results to Indonesian women. Additionally, weighting data were applied in the analyses process in order to adjust the non-proportional sample techniques. Moreover, this survey was conducted using a standard method of data collection from demographic and health surveys as well as the training program for fieldworkers (interviewers, editors, and supervisors) to ensure the success of the survey and to obtain high quality data. The response rate of this survey was high (98\%). However, this study has certain limitations. The study was based on information provided by mothers about their experience of breastfeeding in the last 6 months that is subject to recall bias. Because the study design is cross-sectional, it cannot determine the cause-and-effect relationship between outcome and predictors.

Future studies are needed to analyze the influence of cultural perceptions and traditional practices on EBF across communities in Indonesia. This can help explore the local variation of EBF practices based on local wisdom/culture, women's belief on EBF, and family attitudes in supporting EBF.

In conclusion, this study suggests that working women are at risk for discontinuation of EBF. Mothers who have normal vaginal delivery and initiate breastfeeding within 1 hour have a higher rate of EBF compared to those who do not initiate breastfeeding. These findings have important implications to develop comprehensive guidance and resources about the importance of EBF for women during the early postpartum period. 


\section{CONFLICT OF INTEREST}

No potential conflict of interest relevant to this article was reported.

\section{ACKNOWLEDGMENTS}

This study used the dataset from the 2017 Indonesia Demographic and Health Survey. The author would like to thank the DHS Program for giving the permission to use the IDHS 2017 data for this analysis.

\section{ORCID}

Maria Gayatri: https://orcid.org/0000-0002-2792-5586

\section{REFERENCES}

1. Hashim S, Ishak A, Muhammad J. Unsuccessful exclusive breastfeeding and associated factors among the healthcare providers in East Coast, Malaysia. Korean J Fam Med 2020;41:416-21.

2. Akachi Y, Steenland M, Fink G. Associations between key intervention coverage and child mortality: an analysis of 241 sub-national regions of sub-Saharan Africa. Int J Epidemiol 2018;47:740-51.

3. World Health Organization. Infant and young child feeding: a tool for assessing national practices, policies and programmes. Geneva: World Health Organization; 2003.

4. Duijts L, Jaddoe VW, Hofman A, Moll HA. Prolonged and exclusive breastfeeding reduces the risk of infectious diseases in infancy. Pediatrics 2010;126:e18-25.

5. Nigatu D, Azage M, Motbainor A. Effect of exclusive breastfeeding cessation time on childhood morbidity and adverse nutritional outcomes in Ethiopia: analysis of the demographic and health surveys. PLoS One 2019;14:e0223379.

6. Kramer MS, Kakuma R. Optimal duration of exclusive breastfeeding. Cochrane Database Syst Rev 2012;2012:CD003517.

7. Seid AM, Yesuf ME, Koye DN. Prevalence of exclusive breastfeeding practices and associated factors among mothers in Bahir Dar city, Northwest Ethiopia: a community based cross-sectional study. Int Breastfeed J 2013;8:14.

8. Bhattacharjee NV, Schaeffer LE, Marczak LB, Ross JM, Swartz SJ, Albright J, et al. Mapping exclusive breastfeeding in Africa between 2000 and 2017. Nat Med 2019;25:1205-12.

9. Al-Sahab B, Lanes A, Feldman M, Tamim H. Prevalence and predictors of 6-month exclusive breastfeeding among Canadian women: a national survey. BMC Pediatr 2010;10:20.

10. Amin T, Hablas H, Al Qader AA. Determinants of initiation and exclusivity of breastfeeding in Al Hassa, Saudi Arabia. Breastfeed Med 2011;6:59-68.

11. Ogbo FA, Eastwood J, Page A, Arora A, McKenzie A, Jalaludin B, et al. Prevalence and determinants of cessation of exclusive breastfeeding in the early postnatal period in Sydney, Australia. Int Breastfeed J 2017;12:16.

12. Februhartanty J, Wibowo Y, Fahmida U, Roshita A. Profiles of eight working mothers who practiced exclusive breastfeeding in Depok, Indonesia. Breastfeed Med 2012;7:54-9.
13. Chekol DA, Biks GA, Gelaw YA, Melsew YA. Exclusive breastfeeding and mothers' employment status in Gondar town, Northwest Ethiopia: a comparative cross-sectional study. Int Breastfeed J 2017;12:27.

14. Dun-Dery EJ, Laar AK. Exclusive breastfeeding among city-dwelling professional working mothers in Ghana. Int Breastfeed J 2016;11:23.

15. Sinha B, Chowdhury R, Sankar MJ, Martines J, Taneja S, Mazumder S, et al. Interventions to improve breastfeeding outcomes: a systematic review and meta-analysis. Acta Paediatr 2015;104:114-34.

16. Tan KL. Factors associated with exclusive breastfeeding among infants under six months of age in peninsular Malaysia. Int Breastfeed J 2011;6:2.

17. Lakati A, Binns C, Stevenson M. Breast-feeding and the working mother in Nairobi. Public Health Nutr 2002;5:715-8.

18. Jama A, Gebreyesus H, Wubayehu T, Gebregyorgis T, Teweldemedhin $\mathrm{M}$, Berhe T, et al. Exclusive breastfeeding for the first six months of life and its associated factors among children age 6-24 months in Burao district, Somaliland. Int Breastfeed J 2020;15:5.

19. Lenja A, Demissie T, Yohannes B, Yohannis M. Determinants of exclusive breastfeeding practice to infants aged less than six months in Offa district, Southern Ethiopia: a cross-sectional study. Int Breastfeed J 2016;11:32.

20. National Population and Family Planning Board; Statistics Indonesia; Ministry of Health; The DHS Program ICF. Indonesia Demographic and Health Survey 2017. Jakarta: National Population and Family Planning Board; 2018.

21. Rutstein SO, Johnson K. DHS comparative reports no. 6: The DHS Wealth Index [Internet]. Calverton (MD): ORC Macro; 2004 [cited 2019 Nov 28]. Available from: https://dhsprogram.com/pubs/pdf/cr6/cr6. pdf.

22. Rutstein SO, Rojas G. Guide to DHS statistics: demographic and health surveys methodology. Calverton (MD): ORC Macro; 2006.

23. United Nations Children's Fund. Infant and young child feeding [Internet]. New York (NY): UNICEF; 2019 [cited 2019 Nov 28]. Available from: https://data.unicef.org/topic/nutrition/infant-and-youngchild-feeding/.

24. World Health Organization. Guideline: protecting, promoting and supporting breastfeeding in facilities providing maternity and newborn services. Geneva: World Health Organization; 2017.

25. Nkoka O, Ntenda PAM, Kanje V, Milanzi EB, Arora A. Determinants of timely initiation of breast milk and exclusive breastfeeding in Malawi: a population-based cross-sectional study. Int Breastfeed J 2019;14:37.

26. Ogbo FA, Dhami MV, Awosemo AO, Olusanya BO, Olusanya J, Osuagwu UL, et al. Regional prevalence and determinants of exclusive breastfeeding in India. Int Breastfeed J 2019;14:20.

27. Tadesse F, Alemayehu Y, Shine S, Asresahegn H, Tadesse T. Exclusive breastfeeding and maternal employment among mothers of infants from three to five months old in the Fafan zone, Somali regional state of Ethiopia: a comparative cross-sectional study. BMC Public Health 2019;19:1015.

28. Monteiro FR, Buccini G, Venancio SI, da Costa T. Influence of maternity leave on exclusive breastfeeding: analysis from two surveys conducted in the federal district of Brazil. J Hum Lact 2019;35:362-70.

29. Rimes KA, Oliveira MIC, Boccolini CS. Maternity leave and exclusive breastfeeding. Rev Saude Publica 2019;53:10.

30. Ahmadi M, Moosavi SM. Evaluation of occupational factors on con- 
tinuation of breastfeeding and formula initiation in employed mothers. Glob J Health Sci 2013;5:166-71.

31. Pemo K, Phillips D, Hutchinson AM. Midwives' perceptions of barriers to exclusive breastfeeding in Bhutan: a qualitative study. Women Birth 2020;33:e377-84.

32. Steurer LM. Maternity leave length and workplace policies' impact on the sustainment of breastfeeding: global perspectives. Public Health Nurs 2017;34:286-94.
33. Blixt I, Johansson M, Hildingsson I, Papoutsi Z, Rubertsson C. Women's advice to healthcare professionals regarding breastfeeding: "offer sensitive individualized breastfeeding support": an interview study. Int Breastfeed J 2019;14:51.

34. Kanhadilok S, McCain NL, McGrath JM, Jallo N, Price SK, Chiaranai C. Factors associated with exclusive breastfeeding through four weeks postpartum in Thai adolescent mothers. J Perinat Educ 2016;25:15061. 